\title{
Closed-form SER and Capacity Expressions for Receive Antenna Selection using Orthogonal Space-Time Block Codes
}

\author{
Khoa T. Phan, Chintha Tellambura, and Trong Duy Ngo \\ Department of Electrical and Computer Engineering \\ University of Alberta \\ Edmonton, AB, Canada T6G 2V4 \\ Email: $\{$ khoa, chintha $\} @$ ece.ualberta.ca
}

\begin{abstract}
In multiple-input multiple-output (MIMO) systems, antenna selection where only a subset of antennas at the transmitter and/or receiver are activated for signal transmission is a practical technique for the realization of diversity benefits. Despite extensive research, closed-form symbol-error-rate (SER) and capacity expressions for MIMO systems employing receive antenna selection (RAS) and orthogonal space-time block codes (OSTBCs) are not available. We thus derive exact closed-form expressions for the SER and capacity of an OSTBC MIMO system when $N$ receive antennas out of total $L_{r}$ antennas are selected at the receiver. Our expressions are valid for a frequency-flat Rayleigh fading MIMO channel and avoid numerical integration methods.
\end{abstract}

\section{INTRODUCTION}

Multiple antennas for transmitting and/or receiving data effectively mitigate fading. Obtaining the benefits of multiple transmit antennas may however require the use of special space-time signaling schemes such as orthogonal space-time block codes (OSTBCs), a class of easily decoded spacetime codes that achieve the full diversity order [1], [2]. The family of OSTBCs simplifies maximum-likelihood decoding by decoupling the vector detection problem into a set of scalar detection problems [3].

A major limiting factor in the deployment of MIMO systems is the cost of multiple radio frequency chains (such as amplifiers, mixers and analog-to-digital converters) at both ends of a wireless link. A powerful solution is to select a subset of the available antennas while keeping the advantages of using all antennas [4]. This results in a limited number of transmit/receive chains are dynamically multiplexed between several transmit/receive antennas. Several RAS algorithms to pick the best antenna subset has been proposed [5], [6]. Performance analysis of space-time coding for both uncorrelated and correlated channels incorporated with receive antenna selection have been reported in [7], [8] where only performance bounds were offered.

The main contribution of this paper is to provide closedform symbol-error-rate (SER) and capacity expressions for uncorrelated Rayleigh fading channels with RAS and OSTBCs. A closed-form capacity expression for transmit an- tenna selection and OSTBCs has been derived in [9]. To the best of our knowledge, although RAS is a well researched topic in which various channel/correlation models have been comprehensively treated, no SER expressions for OSTBC MIMO with RAS are available. To simplify the complexity of evaluating the SER, we also propose a direct and accurate SER approximation based on Gauass-Chebyshev quadrature integration [10]. The resulting formulas avoid the need for lengthly Monte Carlo simulation.

The paper is organized as follows. In the next section, the system model and preliminary analysis are presented. In Section 3, we derive the closed-form SER expression as well as its accurate approximation. The main part of this paper, the closed-form expression for capacity, is derived in Section 4. Section 5 gives the conclusions

Notation: Bold symbols denote matrices or vectors. $(\cdot)^{T}$, $(\cdot)^{H}$ and $(\cdot)^{*}$ denote transpose, conjugate transpose and conjugate, respectively. The set of complex numbers is $\mathcal{C}$, and the set of all complex $K \times 1$ vectors, $M \times N$ matrices are denoted by $\mathcal{C}^{K}, \mathcal{C}^{M \times N}$ respectively. A circularly symmetric complex Gaussian variable with mean $\mu$ and variance $\sigma^{2}$ is denoted by $z \sim \mathcal{C N}\left(\mu, \sigma^{2}\right) .\|\|_{F}^{2}$ stands for the Frobenus norm.

\section{SySTEM MODEL}

We consider a MIMO system in a Rayleigh fading environment with $L_{t}$ transmit and $L_{r}$ receive antennas. Channel state information (CSI) is perfectly available at the receiver, but not at the transmitter. $N$ receive antennas out of $L_{r}$ are selected and activated for the signal reception of OSTBC signal matrices, while the remaining receive antennas are inactive. Let $\widetilde{\mathbf{H}} \in \mathcal{C}^{N \times L_{t}}$ be a submatrix of the channel matrix $\mathbf{H} \in \mathcal{C}^{L_{r} \times L_{t}} . \mathbf{H}=\left[h_{i j}\right]$ where $h_{i j} \sim \mathcal{C N}(0,1)$ is the channel gain between the $i$ th transmit and $j$ th receive antenna. $\widetilde{\mathbf{H}}$ consists of the channel gains for the $N$ selected receive antennas and $L_{t}$ transmit antennas. Suppose that $\mathbf{h}_{j}^{T}$ $\left(j=1,2, \ldots, L_{r}\right)$ are rows of the channel matrix $\mathbf{H}$. The rows are sorted according to their norms and assume that $\left\|\mathbf{h}_{i_{1}}^{T}\right\| \geq \ldots \geq\left\|\mathbf{h}_{i_{L_{t}}}^{T}\right\|$ where $i_{k} \in\left\{1,2, \ldots, L_{r}\right\}$. Thus, $\widetilde{\mathbf{H}}$ 
is defined as

$$
\widetilde{\mathbf{H}}=\left[\mathbf{h}_{i_{1}} \mathbf{h}_{i_{2}} \cdots \mathbf{h}_{i_{N}}\right]^{T} .
$$

As can be seen, with this selection criterion, we actually maximize the total received signal power at the receiver. The received signals are expressed as

$$
\mathbf{Y}=\sqrt{\frac{E_{s}}{N_{t}}} \widetilde{\mathbf{H}} \mathbf{X}+\mathbf{V}
$$

where $\mathbf{Y} \in \mathcal{C}^{N \times T}$ is the complex received signal matrix and $\mathbf{X} \in \mathcal{C}^{L_{t} \times T}$ is the complex transmitted signal matrix, which is a member of an OSTBC [2]. $\mathbf{V} \in \mathcal{C}^{N \times T}$ is the additive noise matrix with independent and identical distributed entries of $\mathcal{C N}\left(0, N_{0}\right)$. The coefficient $\sqrt{E_{s} / N_{t}}$ ensures that the total transmitted power in each channel use is $E_{s}$ and independent of number of transmit antennas.

We denote the vector consisting of complex informationbearing symbols prior to space-time encoding as $\mathbf{s}=$ $\left[s_{1}, s_{2}, \ldots, s_{Q}\right]^{T}$ of size $Q \times 1$. Different signal constellations such as M-PAM, M-QAM, or M-PSK can be used with normalization such that $\mathbb{E}\left(\left|s_{i}\right|^{2}\right)=1$. A OSTBC codematrix $\mathbf{X}(\mathbf{s})$ of size $N_{t} \times T$ is then used to encode the input vector with the properties [2]

- All the elements in $\mathbf{X}(\mathbf{s})$ are linear functions of $s_{1}, s_{2}, \ldots, s_{Q}$ and their complex conjugates.

- $\mathbf{X}(\mathbf{s})^{H} \mathbf{X}(\mathbf{s})=\|\mathbf{s}\|_{F}^{2} \mathbf{I}$ for all $\mathbf{s} \in \mathbb{C}^{Q}$.

Since $T$ symbol periods are necessary to transmit $Q$ symbols, the symbol rate $R_{s}$ of the OSTBC is defined as $R_{s}=Q / T$.

When an OSTBC is used, it has been shown that the ML decoder for an OSTBC modulated signal decomposes the MIMO system as $Q$ independent single input single output (SISO) additive white Gaussian noise channels defined as [2]

$$
\tilde{s}_{q}=\sqrt{\frac{E_{s}}{N_{t}}}\left(\frac{1}{R_{s}}\|\widetilde{\mathbf{H}}\|_{F}^{2}\right) s_{q}+\nu_{q}, \quad q=1, \ldots, Q
$$

where $\nu_{q} \sim \mathcal{C N}\left(0, \frac{1}{R_{s}}\|\widetilde{\mathbf{H}}\|_{F}^{2} N_{0}\right)$. We conclude that the achievable SNR per symbol in $M$-ary constellation is

$$
\gamma_{s}=\frac{E_{s}}{N_{0}} \frac{1}{R_{s} N_{t}}\|\widetilde{\mathbf{H}}\|_{F}^{2}=c \rho\|\widetilde{\mathbf{H}}\|_{F}^{2}
$$

where $\rho=\frac{E_{s}}{N_{0}}$ is the SNR per channel use and $c=1 /\left(R_{s} N_{t}\right)$. Therefore, the antenna selection criterion in (1), which selects $N$ receive antennas, maximizes the instantaneous SNR and thereupon minimizes the error rate and maximizes channel capacity.

Let $\gamma_{k}=c \rho\left\|\mathbf{h}_{k}^{T}\right\|^{2}, k=1,2, \ldots, L_{r}$ be the scaled norms of the rows of $\mathbf{H}$. Therefore, $\gamma_{k}$ is a chi-squared i.i.d. random variable with the pdf given by

$$
p_{\gamma_{k}}\left(\gamma_{k}\right)=\frac{\gamma_{k}^{L_{t}-1}}{(c \rho)^{L_{t}}\left(L_{t}-1\right) !} e^{-\gamma_{k} / c \rho} .
$$

In receive antenna selection (1), the best $N$ antennas with the largest $\gamma_{k}$ are selected. Thus, the received SNR per symbol (4) can be written as

$$
\gamma_{s}=\sum_{k=1}^{N} \gamma_{(k)}
$$

where $\gamma_{(k)}=c \rho\left\|\mathbf{h}_{i_{k}}^{T}\right\|^{2}$. The moment generating function (MGF) of $\gamma_{s}$ is given by [11]

$$
\begin{aligned}
\Phi_{\gamma_{s}}(s)= & N\left(\begin{array}{c}
L_{r} \\
N
\end{array}\right) \frac{(c \rho)^{-L_{t} N}}{\left[\left(L_{t}-1\right) !\right]^{N}} \sum_{i_{1}, \ldots, i_{N}} a\left(L_{t} ; i_{1}, \ldots, i_{N}\right) \\
& \prod_{k=1}^{N-1} \frac{i_{k} !}{k^{i_{k}}} \sum_{j=0}^{L_{r}-N}\left(\begin{array}{c}
L_{r}-N \\
j
\end{array}\right)(-1)^{j} \\
& \times\left\{\sum_{n \in B}\left(\begin{array}{c}
j \\
n_{0}, \ldots, n_{L_{t}-1}
\end{array}\right) \frac{\left(c_{n j}+i_{N}\right) !(c \rho)^{r+N+i_{N}}}{(N+j)^{c_{n j}+i_{N}+1} A_{n j}}\right. \\
& \left.\frac{1}{(1+c \rho s)^{r+N-1}} \cdot \frac{1}{\left(1+\frac{N c \rho}{N+j} s\right)^{c_{n j}+i_{N}+1}}\right\}
\end{aligned}
$$

where $\left(\begin{array}{c}j \\ n_{0}, \ldots, n_{L_{t}-1}\end{array}\right)=\frac{j !}{n_{0} ! \cdots n_{L_{t}-1} !}, a\left(L_{t} ; i_{1}, \ldots, i_{N}\right)$ is the coefficient of $x_{1}^{i_{1}} \ldots x_{N}^{i_{N}}$ in expression

$$
\left(x_{1}+x_{2}+\cdots+x_{N}\right)^{L_{t}-1}\left(x_{2}+\cdots+x_{N}\right)^{L_{t}-1} \cdots x_{N}^{L_{t}-1}
$$

and $B$ is the set of all combinations of nonnegative integers of $n_{0}, n_{1}, \ldots, n_{L_{t}-1}$ such that $\sum_{k=0}^{L_{t}-1} n_{k}=j, c_{n j}=\sum_{k=1}^{L_{t}-1} k n_{k}$, $A_{n j}=\prod_{k=2}^{L_{t}-1}(k !)^{n_{k}}$ and $r=\sum_{k=1}^{N-1} i_{k}$. Note that the MGF of $\gamma_{s}$ is the Laplace transform of $f_{\gamma_{s}}(\lambda)$ evaluated at $-s$.

\section{CLOSED-FORM SER EXPRESSIONS}

\section{A. Exact SER for M-ary PAM}

In this section, we derive the closed-form expression for exact average SER with OSTBCs and RAS. In [8], the authors only provided performance bounds. For simplicity, we consider PAM modulation. We have the SER is given as following [12]

$$
\mathrm{SER}_{\gamma_{\mathrm{s}}}=\frac{2}{\pi} \frac{M-1}{M} \int_{0}^{\frac{\pi}{2}} \Phi_{\gamma_{s}}\left(\frac{g_{\text {pam }}}{\sin ^{2} \phi}\right) d \phi
$$

where $g_{\text {pam }}=\frac{3}{M^{2}-1}$. In order to evaluate the exact SER, we define

$I\left(c_{1}, c_{2} ; m_{1}, m_{2}\right)=\frac{1}{\pi} \int_{0}^{\frac{\pi}{2}}\left(\frac{\sin ^{2} \phi}{\sin ^{2} \phi+c_{1}}\right)^{m_{1}}\left(\frac{\sin ^{2} \phi}{\sin ^{2} \phi+c_{2}}\right)^{m_{2}} d \phi$

for which a closed-form expression is available in [12]. However, for clarity, we restate the result as following

$$
\begin{aligned}
I\left(c_{1}, c_{2} ; m_{1}, m_{2}\right)= & \frac{\left(\frac{c_{1}}{c_{2}}\right)^{m_{2}-1}}{2\left(1-\frac{c_{1}}{c_{2}}\right)^{m_{1}+m_{2}-1}}\left[\sum_{k=0}^{m_{2}-1}\left(\frac{c_{2}}{c_{1}}-1\right)^{k} B_{k}\right. \\
& \left.I_{k}\left(c_{2}\right)-\frac{c_{1}}{c_{2}} \sum_{k=0}^{m_{1}-1}\left(1-\frac{c_{1}}{c_{2}}\right)^{k} C_{k} I_{k}\left(c_{1}\right)\right]
\end{aligned}
$$

where

$$
\begin{gathered}
B_{k} \triangleq \frac{A_{k}}{\left(\begin{array}{c}
m_{1}+m_{2}-1 \\
k
\end{array}\right)}, \quad C_{k} \triangleq \sum_{n=0}^{m_{2}-1} \frac{\left(\begin{array}{l}
k \\
n
\end{array}\right)}{\left(\begin{array}{c}
m_{1}+m_{2}-1 \\
n
\end{array}\right)} A_{n}, \\
A_{k} \triangleq(-1)^{m_{2}-1+k} \frac{\left(\begin{array}{c}
m_{2}-1 \\
k
\end{array}\right)}{\left(m_{2}-1\right) !} \prod_{\substack{n=1 \\
n \neq k+1}}^{m_{2}}\left(m_{1}+m_{2}-n\right),
\end{gathered}
$$




$$
\begin{aligned}
\operatorname{SER}_{\gamma_{\mathrm{s}}}= & \frac{2(M-1) N\left(\begin{array}{c}
L_{r} \\
N
\end{array}\right)(c \rho)^{-L_{t} N}}{M\left[\left(L_{t}-1\right) !\right]^{N}} \sum_{i_{1}, \ldots, i_{N}} a\left(L_{t} ; i_{1}, \ldots, i_{N}\right) \prod_{k=1}^{N-1} \frac{i_{k} !}{k^{i_{k}}} \sum_{j=0}^{L_{r}-N}\left(\begin{array}{c}
L_{r}-N \\
j
\end{array}\right)(-1)^{j} \\
& \times\left\{\sum_{n \in B}\left(\begin{array}{c}
j \\
n_{0}, \ldots, n_{L_{t}-1}
\end{array}\right) \frac{\left(c_{n j}+i_{N}\right) !(c \rho)^{r+N+i_{N}}}{(N+j)^{c_{n j}+i_{N}+1} A_{n j}} I\left(c \rho g_{\text {pam }}, \frac{N c \rho g_{\text {pam }}}{N+j} ; r+N-1, c_{n j}+i_{N}+1\right)\right\} .
\end{aligned}
$$

and

$$
I_{k}(c)=1-\sqrt{\frac{c}{1+c}}\left[1+\sum_{n=1}^{k} \frac{(2 n-1) ! !}{n ! 2^{n}(1+n)^{n}}\right]
$$

with the double factorial notation denoting the product of only odd integers from 1 to $2 k-1$. Then, the SER is given by the closed-form formula (13).

Although in closed-form, the SER expression (13) appears to be complicated. Therefore, we now provide an accurate approximation for (8) based on the Chebyshev polynomials. By changing variable $t=\sin \phi$, the integral formula (8) can be written in terms of new variable as

$$
\mathrm{SER}_{\gamma_{\mathrm{s}}}=\frac{2}{\pi} \frac{M-1}{M} \int_{0}^{1} \frac{\Phi_{\gamma_{s}}\left(\frac{g_{\text {pam }}}{t^{2}}\right)}{\sqrt{1-t^{2}}} d t
$$

whose the integrand is an even function w.r.t variable $t$. Therefore, we can evaluate it alternatively as following

$$
\mathrm{SER}_{\gamma_{\mathrm{s}}}=\frac{1}{\pi} \frac{M-1}{M} \int_{-1}^{1} \frac{\Phi_{\gamma_{s}}\left(\frac{g_{\text {pam }}}{t^{2}}\right)}{\sqrt{1-t^{2}}} d t .
$$

With this form, we can readily apply the result in [10], name the Chebyshev polynomials of first kind

$$
\mathrm{SER}_{\gamma_{\mathrm{s}}}=\frac{1}{\pi} \frac{M-1}{M} \sum_{j=1}^{n} w_{j} \Phi_{\gamma_{s}}\left(\frac{g_{\text {pam }}}{t_{j}^{2}}\right)+R_{n},
$$

where the coefficients are given by

$$
w_{j}=\frac{\pi}{n}, \quad t_{j}=\cos \frac{(2 j-1) \pi}{2 n} .
$$

and the remainder, which will be ignored for computation, has the following form

$$
R_{n}=\frac{\pi}{(2 n) ! 2^{2 n-1}} f^{(2 n)}(\xi), \quad(-1 \leq \xi \leq 1) .
$$

The larger the value of $n$ will provide better approximation for (8).

\section{B. Exact SER for M-ary QAM}

Similar approach can be used to compute the SER for QAM modulation (as well as M-PSK). Given the MGF of the SNR per symbol, the SER for QAM-modulation is given by [12]

$$
\begin{aligned}
\operatorname{SER}_{\gamma_{\mathrm{s}}} & =\frac{4}{\pi}\left(\frac{\sqrt{M}-1}{\sqrt{M}}\right) \int_{0}^{\frac{\pi}{2}} \Phi_{\gamma_{s}}\left(\frac{g_{\text {qam }}}{\sin ^{2} \phi}\right) d \phi \\
& -\frac{4}{\pi}\left(\frac{\sqrt{M}-1}{\sqrt{M}}\right)^{2} \int_{0}^{\frac{\pi}{4}} \Phi_{\gamma_{s}}\left(\frac{g_{\text {qam }}}{\sin ^{2} \phi}\right) d \phi
\end{aligned}
$$

where $g_{\text {qam }}=\frac{3}{2(M-1)}$. The first term can be computed similarly as for the case of PAM. To compute the second term, we define

$$
J(c, m)=\frac{1}{\pi} \int_{0}^{\frac{\pi}{4}}\left(\frac{\sin ^{2} \phi}{\sin ^{2} \phi+c}\right)^{m} d \phi
$$

whose closed-form expression is provided in [12]. In order to use the above form, we need to do partial fraction for the MGF in (7). This will be explained in the next section where we derive the closed-form expression for capacity.

\section{CLOSED-FORM CAPACITY FORMULA}

In order to evaluate the average channel capacity, we need the probability density function of $\gamma_{s}, f_{\gamma_{s}}(\lambda)$ which in turn can be obtained by doing the inverse Laplace transform of (7). Using partial fraction, we have the following result

$$
\begin{gathered}
\varphi(s)=\frac{1}{(1+c \rho s)^{r+N-1}} \cdot \frac{1}{\left(1+\frac{N c \rho}{N+j} s\right)^{c_{n j}+i_{N}+1}} \\
=\sum_{m=1}^{r+N-1} K_{m} \Psi_{c \rho}^{m}(s)+\sum_{n=1}^{c_{n j}+i_{N}+1} \tilde{K}_{n} \Psi_{\frac{N c \rho}{N+j}}^{n}(s)
\end{gathered}
$$

where $\Psi_{\gamma}(s)=(1+\gamma s)^{-1}$ and the coefficients are given by

$K_{m}=\frac{(c \rho)^{m+1-r-N}}{(r+N-1-m) !} \cdot \frac{\partial^{r+N-1-m}}{\partial s^{r+N-1-m}}\left[\Psi_{\frac{N c \rho}{N+j}}^{c_{n j}+i_{N}+1}(s)\right]_{s=-\frac{1}{c \rho}}$

and

$\tilde{K}_{n}=\frac{\left(\frac{N c \rho}{N+j}\right)^{n-c_{n j}-i_{N}-1}}{\left(c_{n j}+i_{N}+1-n\right) !} \cdot \frac{\partial^{c_{n j}+i_{N}+1-n}}{\partial s^{c_{n j}+i_{N}+1-n}}\left[\Psi_{c \rho}^{r+N-1}(s)\right]_{s=-\frac{N+j}{N c \rho}}$

We are ready to take the inverse Laplace transform of $\Phi_{\gamma_{s}}(s)$, using the linearity property and the fact that

$$
\mathcal{L}^{-1}\left\{\Psi_{\gamma}^{m}(s)\right\}=\frac{1}{(m-1) ! \gamma^{m}} \lambda^{m-1} e^{-\frac{\lambda}{\gamma}} .
$$

The pdf of $\gamma_{s}=c \rho\|\widetilde{\mathbf{H}}\|_{F}^{2}$ is obtained in (25). Then, the exact average capacity in nats/s/Hz is given by (26), where we use the following result from [13, App. B]

$$
\begin{aligned}
\hat{C}_{m-1}(\nu) & \triangleq \int_{0}^{\infty} \log (1+\lambda) \lambda^{m-1} e^{-\nu \lambda} d \lambda, \nu>0, n=1,2, \ldots \\
& =(m-1) ! e^{\nu} \sum_{k=1}^{m} \frac{\Gamma(-m+k, \nu)}{\nu^{k}}
\end{aligned}
$$

where $\Gamma(a, z)=\int_{z}^{\infty} e^{-x} x^{a-1} d x$ is the complementary incomplete gamma function. 


$$
\begin{aligned}
& f_{\gamma_{s}}(\lambda)=\mathcal{L}^{-1}\{\Phi(s)\} \\
&=N\left(\begin{array}{c}
L_{r} \\
N
\end{array}\right) \frac{(c \rho)^{-L_{t} N}}{\left[\left(L_{t}-1\right) !\right]^{N}} \sum_{i_{1}, \ldots, i_{N}} a\left(L_{t} ; i_{1}, \ldots, i_{N}\right) \prod_{k=1}^{N-1} \frac{i_{k} !}{k^{i_{k}}} \sum_{j=0}^{L_{r}-N}\left(\begin{array}{c}
L_{r}-N \\
j
\end{array}\right)(-1)^{j} \\
& \times\left\{\sum_{n \in B}\left(\begin{array}{c}
j \\
n_{0}, \ldots, n_{L_{t}-1}
\end{array}\right) \frac{\left(c_{n j}+i_{N}\right) !(c \rho)^{r+N+i_{N}}}{(N+j)^{c_{n j}+i_{N}+1} A_{n j}}\right. \\
&\left.\left\{\sum_{m=1}^{r+N-1} \frac{K_{m}}{(m-1) !(c \rho)^{m}} \lambda^{m-1} e^{-\frac{\lambda}{c_{\rho}}}+\sum_{n=1}^{c_{n j}+i_{N}+1} \frac{\tilde{K}_{n}}{(n-1) !\left(\frac{N c \rho}{N+j}\right)^{n}} \lambda^{n-1} e^{-\frac{\lambda}{N c \rho}}\right\}\right\}
\end{aligned}
$$

$$
\begin{aligned}
& C= \mathbb{E}\left\{R_{s} \log \left(1+\gamma_{s}\right)\right\} \\
&=R_{s} \int_{0}^{\infty} \log (1+\lambda) f_{\gamma_{s}}(\lambda) d \lambda \\
&=R_{s} N\left(\begin{array}{c}
L_{r} \\
N
\end{array}\right) \frac{(c \rho)^{-L_{t} N}}{\left[\left(L_{t}-1\right) !\right]^{N}} \sum_{i_{1}, \ldots, i_{N}} a\left(L_{t} ; i_{1}, \ldots, i_{N}\right) \\
& \prod_{k=1}^{N-1} \frac{i_{k} !}{k^{i_{k}}} \sum_{j=0}^{L_{r}-N}\left(\begin{array}{c}
L_{r}-N \\
j
\end{array}\right)(-1)^{j} \\
& \times\left\{\sum_{n \in B}\left(\begin{array}{c}
j \\
n_{0}, \ldots, n_{L_{t}-1}
\end{array}\right) \frac{\left(c_{n j}+i_{N}\right) !(c \rho)^{r+N+i_{N}}}{(N+j)^{c_{n j}+i_{N}+1} A_{n j}}\right. \\
&\left.\left\{\sum_{m=1}^{r+N-1} \frac{K_{m}}{(m-1) !(c \rho)^{m}} \hat{C}_{m-1}(1 / c \rho)+\sum_{n=1}^{c_{n j}+i_{N}+1} \frac{\tilde{K}_{n}}{(n-1) !\left(\frac{N c \rho}{N+j}\right)^{n}} \hat{C}_{n-1}\left(\frac{N+j}{N c \rho}\right)\right\}\right\}
\end{aligned}
$$

The SER expression in (13), its approximation in (15), and the capacity formula (26) do not involve any integrations which could be computed easily with precise accuracy using mathematical softwares, such as MATLAB.

\section{CONCLUSION}

In this paper, we have analyzed the performance and capacity of RAS and OSTBCs for MIMO systems. We use the approach via the method of MFG of the system's effective SNR. First using the partial fraction and then inverse Laplace transform, the probability density function of the SNR is obtianed. Then, the closed-form SER for standard constellations and capacity expressions are derived. An accurate approximation of SER expression is also provided based on the Chebyshev polynomials of first kind. Our results are sufficiently general to handle an arbitrary number of antennas.

\section{REFERENCES}

[1] S. Alamouti, "A simple transmit diversity technique for wireless communications," IEEE J. Select. Areas Commun., vol. 16, pp. 1451 - 1458, Oct. 1998.

[2] V. Tarokh, H. Jafarkhani, and A. Calderbank, "Space-time block codes from orthogonal designs," IEEE Trans. Inform. Theory, vol. 45, no. 5, pp. 1456 - 1467, July 1999.

[3] E. G. Larsson and P. Stoica, Space-time Block Coding for Wireless Communications. Cambridge University Press, 2003.
[4] A. Gorokhov, D. Gore, and A. Paulraj, "Performance bounds for antenna selection in MIMO systems," in Proc. IEEE Int. Conf. Communications (ICC), Anchorage,USA, 2003, pp. 3021-3025.

[5] A. Dhananjay, A. Gore, and A. Paulraj, "Receiver antenna selection for MIMO spatial multiplexing: theory and algorithms," IEEE Trans. Signal Processing, vol. 51, no. 11, pp. 2796 - 2807, Nov. 2003.

[6] K. T. Phan and C. Tellambura, "Union bound based receive antenna selection in MIMO spatial multiplexing using convex optimization," Accepted IEEE Signal Processing Lett.

[7] D. Gore and A. Paulraj, "MIMO antenna subset selection with spacetime coding," IEEE Trans. Signal Processing, vol. 50, no. 10, pp. 25802588, Oct. 2002.

[8] X. N. Zeng and A. Ghrayeb, "Performance bounds for space-time block codes with receive antenna selection," IEEE Trans. Inform. Theory, vol. 50, no. 9, pp. 2130 - 2137, Sept. 2004.

[9] K. T. Phan and C. Tellambura, "Capacity analysis for transmit antenna selection using orthogonal space-time block codes," Submitted IEEE Commun. Lett.

[10] M. Abramowitz and I. A. Stegun, Handbook of Mathematical Functions with Formulas, Graphs, and MathematicalTables. New York: Dover, 1972.

[11] Y. Ma and C. C. Chai, "Unified error probability analysis for generalized selection combining inNakagami fading channels," IEEE J. Select. Areas Commun., vol. 18, no. 11, pp. 2198 - 2210, Nov. 2000.

[12] M. K. Simon and M.-S. Alouini, Digital Communication over Fading Channels: A Unified approach to perfromance analysis, 1st ed. New York: Wiley Series in Telecommunications and Signal Processing, 2000.

[13] M.-S. Alouini and A. J. Goldsmith, "Capacity of Rayleigh fading channels under different adaptive transmissionand diversity-combining techniques," IEEE Trans. Veh. Technol., vol. 48, pp. 1165-1181, July 1999. 